\title{
Portales para la comunicación estratégica de las instituciones políticas
}

\author{
Victoria Martínez Navarro \\ $\mathrm{M}^{\mathrm{a}}$ Dolores Ayuso García ${ }^{* *}$
}

\section{Resumen}

Este artículo es una aproximación al estudio de las fuentes de información para la comunicación estratégica de las instituciones políticas. Con este objetivo las autoras presentan a la comunidad científica el escenario de trabajo de una investigación en curso, tendente a definir la panorámica de las fuentes de información ligadas al área de relaciones informativas y comunicativas de los ministerios del Estado Español. Mediante una revisión de las sedes web de prensa se define la tipología de las fuentes, se establece el nivel de desarrollo que presentan, y se concluye en su papel de intérpretes del proyecto institucional del gobierno.

Palabras clave: Fuentes de información. Evaluación de la internet. Valor estratégico de la información. Comunicación estratégica. Comunicación política.

Título: Portais para comunicação estratégica das instituições políticas.

\section{Resumo}

Este artigo é uma abordagem para o estudo das fontes de informação para comunicação estratégica das instituições políticas. Com este objetivo, as autoras apresentam para comunidade científica a fase da investigação em curso destinada a definir o panorama das fontes de informação relacionadas com a área de informação e comunicação dos ministérios do Estado Espanhol. Mediante analise dos sites da imprensa pode-se definir a tipologia das fontes, estabelecer o nível de desenvolvimento que apresentam e se concluir seu papel como interpretes do projeto institucional do governo.

Palavras-chave: Fontes de informação. Evolução da internet. Valor estratégico da informação. Comunicação estratégica. Comunicação política.

\footnotetext{
* Profesora asociada. Facultad de Comunicación y Documentación. Universidad de Murcia. España. vicmarti@um.es
} 
Title: Portals for strategic communication of political institutions.

\section{Abstract}

This article is an approach to the study of the sources of information for decisionmaking on communication strategies on the part of the political institutions. With this aim, the authors present to the scientific community the piece of work of an ongoing investigation, which seeks to define the overview of the sources of information related to the area of information and communication links of the departments of the Spanish State. Through a review of the websites of the press the types of sources are defined. The level of development is stablished, as presented and concluded in its role as interpreters of institutional project of the government in power.

Keywords: Information sources. Evaluation internet. Strategic value of information. Strategic communication. Communication policy.

\section{Introducción.}

En los últimos tiempos estamos asistiendo a un importante cambio en la forma de competir de las organizaciones, debido a asuntos tales como la globalización, el aumento de la competitividad empresarial, la existencia de un consumidor cada vez más exigente o los vertiginosos cambios del entorno en el que las empresas operan. Dado este panorama, las organizaciones gubernamentales deben buscar nuevas fuentes de ventaja competitiva e intentar orientarse hacia las percepciones que los ciudadanos tienen de un "buen gobierno". Los portales de las instituciones políticas se han convertido en herramientas claves en los planes de comunicación institucional, desde éstos se cuenta qué hace el equipo de gobierno, creando reputación de marca, y buscando una mayor proyección social. Ante esta situación, las instituciones políticas requieren guías o modelos que les orienten sobre como competir en este ambiente complejo, pero respetando sus características organizativas particulares, es decir, sus servicios y sus fines no lucrativos. En estas circunstancias, encontramos que la comunicación corporativa o institucional se convierte en una herramienta estratégica y competitiva no sólo porque ayuda a formar la imagen deseada en los mercados sino porque nos permite influir sobre ellos.

Según lo anterior, el objetivo de este artículo es el estudio de los modelos de comunicación estratégica de las instituciones políticas del Estado Español. Dicho estudio se aborda desde el análisis de las sedes web ligadas al área de relaciones informativas y comunicativas de los ministerios, al objeto de definir la tipología de fuentes de información 
Portales para la comunicación estratégica de las instituciones políticas

que ofrecen, su nivel de desarrollo y su papel como intérpretes del proyecto institucional del gobierno de la Administración General del Estado.

Uno de los factores críticos para el buen desempeño de la labor de comunicación en una institución política consiste en lograr que su oferta de valor sea percibida en forma positiva por su público objetivo. No importa cómo midamos la función objetiva de la institución política, en este caso de los ministerios, pues su éxito radicará en las percepciones que se formen de sus productos comunicativos. En este escenario comunicacional, los portales web de los ministerios se mantienen a salvaguarda de la lucha diaria por las preferencias del mercado, y se convierten así en una herramienta clave en la política de comunicación de la institución.

Este estudio se enmarca dentro de una línea de investigación que trata de analizar el papel de las fuentes de información ligadas al área de la comunicación estratégica de las instituciones políticas. Los hallazgos y conclusiones que obtengamos en este trabajo esperamos que puedan servir de base para futuras investigaciones relacionadas con las fuentes de información para la toma de decisiones estratégicas, con independencia del ámbito geográfico e institucional que abordaremos.

\section{La nueva configuración del mensaje gubernamental.}

Si pensamos que podemos asistir a la rueda de prensa del Consejo de Ministros desde casa o desde nuestro puesto de trabajo, sin necesidad de desplazarnos hasta La Moncloa, nos damos cuenta de que nada impide que esta información política este a nuestra disposición en tiempo real. Desde hace unos años la presencia de la información de actualidad política en la Red ha abierto un nuevo canal de comunicación desde los poderes públicos hasta el ciudadano, y en éste se ha forjado una nueva configuración del mensaje gubernamental.

El discurso del presidente del Gobierno, del portavoz, de un ministro, o de un parlamentario que habla desde su escaño no solamente se ofrece a los medios de comunicación habituales (prensa, radio y televisión), ahora se ofrece también - sin mediación alguna - a los ciudadanos, estudiosos o investigadores de la materia que disponen de un

acceso más directo e inmediato al mensaje gubernamental. El papel privilegiado que hasta ahora venían teniendo los medios de comunicación tradicionales y las propias publicaciones oficiales - en especial los boletines y diarios de sesiones parlamentarios - viene a ser redefinido con la presencia de los actos informativos de los poderes públicos en las web gubernamentales. 
Con esta perspectiva, podemos afirmar que la nueva configuración del mensaje gubernamental presta gran importancia a la dimensión personal. Es decir, a la representación en una persona del gobierno del mensaje institucional. De ahí, tal y como mostrará esta investigación, la importancia de los contenidos periodísticos en los primeros niveles de los portales web ministeriales, y será una constante en todos ellos la información de actualidad de la agenda de los altos cargos ministeriales.

Se comprende así la necesidad de formular de nuevo la tarea que organiza la publicación y difusión de la información periodística de actualidad desde el poder gubernamental hacia los ciudadanos. Esta nueva configuración del mensaje gubernamental enlaza con la política de la reutilización de la información del sector público, pues toda información generada por los poderes públicos ha de ser difundida a los ciudadanos, no solamente como medida de transparencia administrativa, sino más bien - para el caso que nos ocupa - como instrumento para potenciar la confianza de los ciudadanos en sus gobernantes. De ahí que sean características propias de las fuentes informativas de las sedes web de los gabinetes de prensa gubernamentales: la información completa, accesible, con una difusión proactiva, de acceso por canales individuales de comunicación como Internet, relevante, fácil de encontrar y de usar, integrada y organizada estratégicamente en el marco del portal gubernamental.

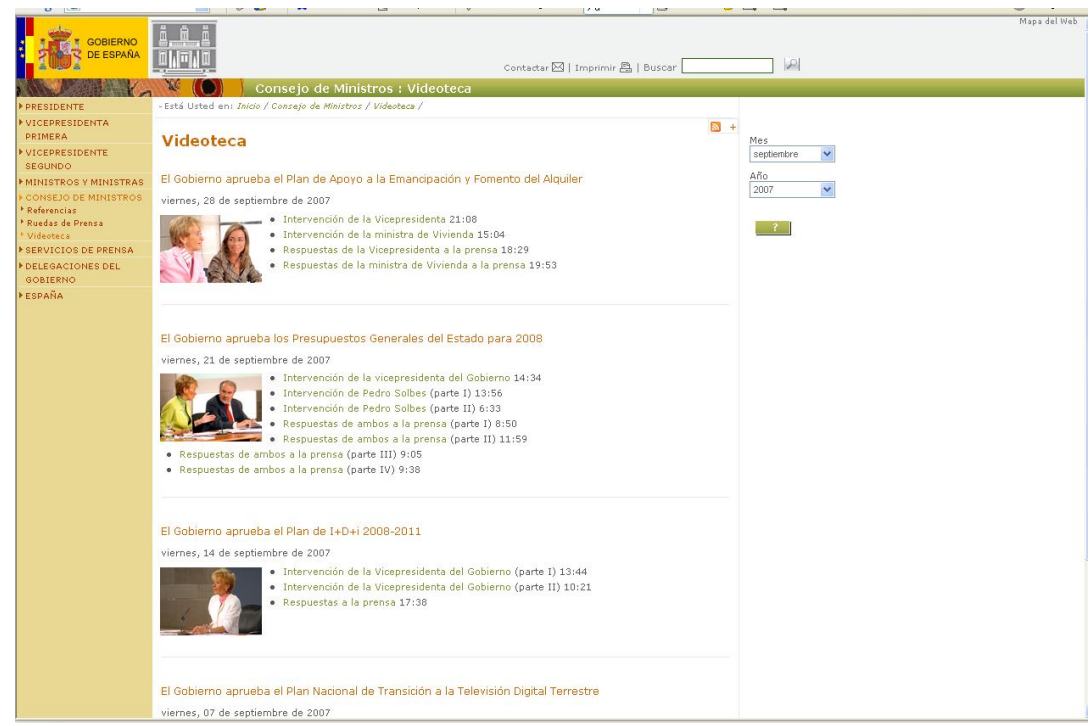

Figura 1. Sección Videoteca. La Moncloa.

Fuente: Web de La Moncloa. Gobierno de España. 
Portales para la comunicación estratégica de las instituciones políticas

El sector público es un importante mercado de contenidos. La organización estratégica en la web instituticional de un gobierno es consecuencia de la creciente eficiencia de la información periodística de actualidad política. Ésta tras su tratamiento por las tecnologías de la información mejora su capacidad para ser utilizada por empresas y ciudadanos con fines privados.

Estos contenidos ya tienen presencia en la Internet móvil. Sirva de ejemplo, la presidencia del Gobierno de España que desde 2007 dispone de la versión para dispositivos móviles de su web oficial con el fin de hacer su portal web más versátil, accesible y, en definitiva, más útil y cercano a todos los ciudadanos. Los profesionales de la información disponen así de una versión ligera de la web de La Moncloa ${ }^{1}$, que está preparada para dispositivos tipo PDA y teléfonos móviles con navegador. Los contenidos movilizados son:

a) Home: noticias destacadas y otras noticias;

b) Presidente: biografía, discurso de investidura, intervenciones, actividades internacionales, actividades nacionales, fototeca, relación anteriores Presidentes;

c) Consejo de Ministros: referencias y ruedas de prensa;

d) Servicios de prensa: notas de prensa, boletín diario de prensa nacional, boletín de prensa internacional, Agenda de la comunicación, Consejerías de información, corresponsales de medios extranjeros, acreditaciones para actos internacionales, fototeca.

\section{Metodología.}

El trabajo mide el grado de estandarización informativa de las sedes web de los gabinetes de prensa ministeriales como indicador de calidad de sus contenidos, y como instrumento clave para la comunicación estratégica de la institución gubernamental. El método empleado se sirve del protocolo de evaluación de las autoras "Protocolo para la evaluación de fuentes y recursos informativos en la sociedad del conocimiento",2. El Protocolo cuenta con un programa de auditoria que ha sido empleado por las autoras en

\footnotetext{
${ }^{1}$ La Moncloa. [en línea] . [fecha de acceso 15 de noviembre 2007]. Disponible en Internet: <http://movil.lamoncloa.es>.

2 AYUSO GARCÍA, Ma Dolores y MARTÍNEZ NAVARRO, Victoria (2005): Protocolo para la evaluación de fuentes y recursos de información en la Sociedad del Conocimiento. Revista General de Información y Documentación, n. 1, vol. 15, p. 21-53.
} 
trabajos anteriores ${ }^{3}$, y que se enmarca en la actual Sociedad del Conocimiento como una herramienta de calidad y de valor añadido a los servicios de información digital, midiendo la calidad y oportunidad del recurso digital ante un colectivo de profesionales especializados.

La tarea de evaluación requiere una metodología propia, en la que el profesional de la documentación, suma o añade a la ya tradicional identificación y recuperación de información en Internet, una descripción de valor añadido a unos recursos digitales que por su naturaleza requieren de un control documental que ha de considerar necesariamente:

a) Las propiedades o características del recurso digital a evaluar: Parámetros;

b) Los elementos del recurso digital que se van a considerar para cada una de las características anteriores: Indicadores;

c) $\mathrm{Y}$ en tercer lugar aquellos medios por los que se procede a determinar la calidad del recurso digital: Procedimientos.

Como explicamos en el proceso de investigación, a través de la evaluación y el análisis descriptivo plasmaremos el estado general de los portales ministeriales, identificando las diferentes estrategias basadas en la comunicación institucional, para posteriormente observar si hay sectores que de forma mayoritaria se adhieren a una de estas estrategias.

El contexto de presentación de este trabajo de investigación, un artículo científico, obliga a las autoras a ceñirse a unas normas que las llevarán a considerar solamente algunos de los indicadores considerados en el marco global de la investigación en curso. La revisión y recogida de datos ha tenido lugar tras la remodelación del Gobierno de la Administración General del Estado Español de julio de 2007, el anexo I muestra las URLs de las 17 sedes web objeto del estudio: Ministerio de la Presidencia; Ministerio de Economía y Hacienda; Ministerio de Asuntos Exteriores y Cooperación; Ministerio de Justicia; Ministerio de Defensa; Ministerio del Interior; Ministerio de Fomento; Ministerio de Educación, Política Social y Deporte; Ministerio de Trabajo e Inmigración; Ministerio de Industria, Turismo y Comercio; Ministerio de Medio Ambiente, Medio Rural y Marino; Ministerio de

\footnotetext{
${ }^{3}$ AYUSO GARCÍA, Ma Dolores y MARTÍNEZ NAVARRO, Victoria (2006): "Metodología de evaluación de recursos en bibliotecas digitales. Parámetros e indicadores de calidad”. En Ciencias de la Información, n, 1, vol. 17 , pp. $25-44$.

- (2006): "Evaluación de calidad de fuentes y recursos digitales: guía de buenas prácticas". En Anales de Documentación, 9, pp- 17-42.

- (2007): “Alfabetización informacional y servicios de referencia virtual". Actas del Congreso Internacional de la ISKO. León, 18-20 de abril.
} 
Portales para la comunicación estratégica de las instituciones políticas

Administraciones Públicas; Ministerio de Cultura; Ministerio de Sanidad y Consumo; Ministerio de Vivienda; Ministerio de Ciencia e Innovación; Ministerio de Igualdad.

La evaluación de las sedes web comienza con la identificación y exploración de la sede ministerial elegida, pero sin aplicar todavía los indicadores de evaluación. No es necesario recorrer todos los niveles, las tareas de identificación se centrarán en la exploración sistemática de las secciones más importantes, incluyendo siempre aquellas relativas a la autoría y la agenda temática del departamento de comunicación. A continuación, se realiza el escrutinio de cada uno de los indicadores que se evalúan. Si la sede web es muy amplia, la exploración puede limitarse a partes o secciones que sean relativamente autónomas.

El cuestionario de evaluación empleado se centra en aquéllos indicadores que como muestran los siguientes epígrafes explora la visibilidad de la sede web de prensa desde el portal ministerial, la tipología de fuentes de información periodísticas ofrecidas, así como el nivel de desarrollo desde el punto de vista de la recuperabilidad, exhaustividad e hipertextualidad. Características estas últimas que dotan a la sede de calidad documental y permiten que se constituya como fuente de información para la investigación periodística.

\section{Evaluación de las sedes ministeriales.}

El Gobierno del Estado Español, como cualquier institución gubernamental, quiere transmitir a los ciudadanos una imagen positiva, procurando que sus programas y actuaciones se conviertan en un referente social. La misión de la dirección de comunicación será la difusión del conocimiento sobre las distintas actuaciones y programas de la institución de manera interna y externa. En esta labor de difusión se definen diversos escenarios de trabajo ruedas de prensa, comunicados de prensa, campañas publicitarias, etc - que quedan compilados bajo el paraguas de unas fuentes de información que actúan como elementos clave para la comunicación estratégica de la institución gubernamental.

Dichas fuentes de información forman parte del proyecto corporativo y su plan estratégico. O dicho de otro modo, éstas participan en el plan estratégico y lo hacen comunicable, como elementos que son de la estrategia global de comunicación del gobierno. El primer indicador a evaluar la Visibilidad nos permite medir dos elementos clave: 
a) Enlace desde el portal ministerial: mide la presencia o ausencia de un enlace a la sede web de prensa o de comunicación desde el portal o página web principal del ministerio en cuestión.

b) Publicación de contenidos en el portal ministerial: mide la presencia o ausencia de contenidos propios de la sede web de prensa en el portal web. Sirva de muestra, una de las prácticas más habituales de este indicador, como es la publicación en el portal de parte de las notas de prensa o documentos fotográficos de los actos del ministerio en el día en cuestión, así como las campañas publicitarias de las actuaciones gubernamentales más reseñables.

El procedimiento empleado para la evaluación del indicador de la visibilidad ha sido la visita de los portales web de los ministerios, buscando en ellos un enlace a la sede web de prensa, así como la publicación de contenidos de la misma en el propio portal web. Tras la recogida de datos y su procesamiento los resultados obtenidos muestran la importancia que el poder gubernamental otorga a estas fuentes de información, pues el $100 \%$ las sedes web evaluadas cuentan con un enlace directo desde el portal de acceso a la institución. Dicho enlace se presenta bajo diferentes epígrafes del tipo: comunicación, actualidad informativa, prensa, gabinete de comunicación, etc. Y en segundo lugar, también la totalidad de las sedes evaluadas publican contenidos propios de la web de prensa en el portal web del ministerio en cuestión. De esta forma, la información se acerca al ciudadano, que no tendrá que navegar para conocer la actualidad informativa del gobierno. Ésta se ofrece en las sedes ministeriales desde el portal de acceso y ocupando, en la mayoría de los casos, la parte central de la página web. Sirva de buena práctica, el portal web del Ministerio de Defensa que, tal y como muestra la siguiente figura, está diseñado ofreciendo una prioridad absoluta a la actualidad informativa de la institución. Toda la parte central del portal ofrece las últimas previsiones y notas de prensa del Ministerio. 
Portales para la comunicación estratégica de las instituciones políticas

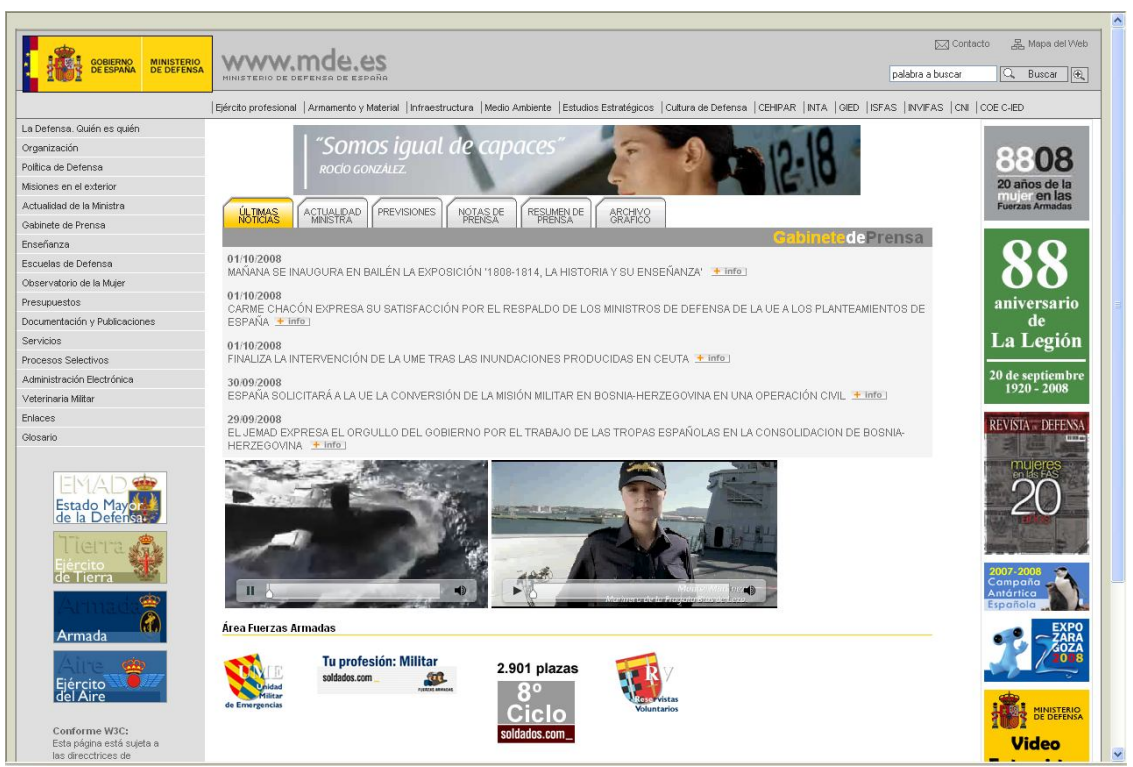

Figura 2. Detalle de publicación de contenidos desde el portal web ministerial.

Fuente: Portal web del Ministerio de Defensa.

El segundo indicador a evaluar es la Tipología de Fuentes. Hemos seleccionado este indicador al objeto de unir el valor documental de toda fuente de información con el propósito de la acción comunicativa gubernamental. La siguiente gráfica muestra, en porcentajes, los tipos de fuentes que revela nuestro estudio.

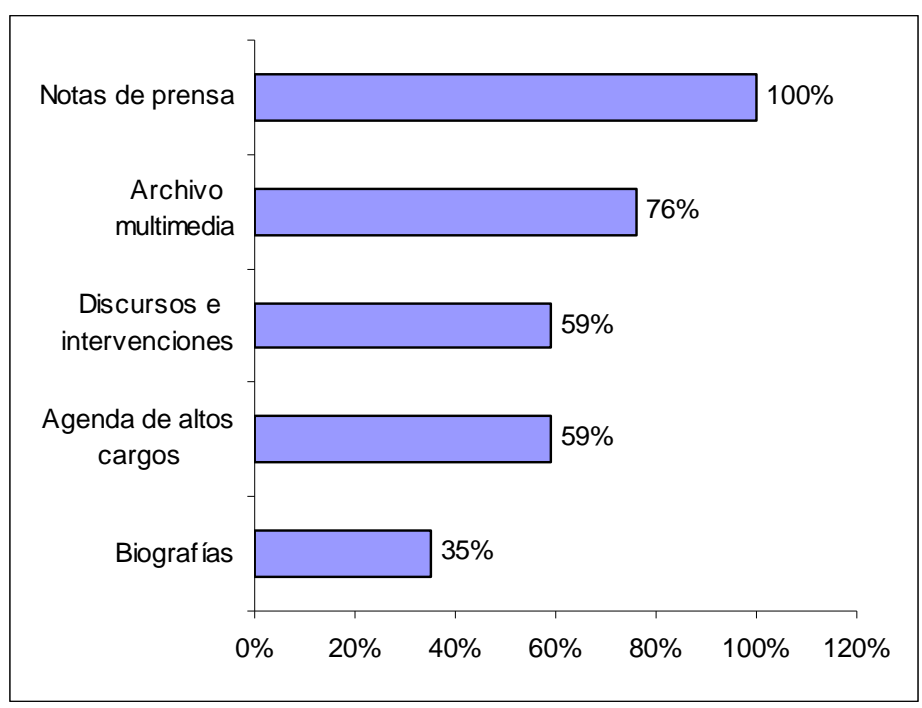

Gráfica 1. Tipología de fuentes de información de las sedes web de prensa.

Fuente: Elaboración propia. 
A este respecto, resulta preciso indicar que se ha llevado a cabo una revisión analítica de cada sede web ministerial, y que los datos ofrecidos han de interpretarse en relación con la utilidad funcional de las fuentes en el sistema de comunicación periodístico. La primacía recae sobre las notas de prensa y el archivo multimedia (fotografías y cortes de voz principalmente), fuentes éstas que suelen documentar los actos institucionales de las agendas de ministros, secretarios de estado y directores generales.

El tercer indicador a evaluar es el Acceso a la Colección Retrospectiva de fuentes de información. Con este indicador, se evalúa la existencia y el nivel de desarrollo de la búsqueda retrospectiva en las fuentes de información que hemos identificado en el indicador anterior, especialmente en notas de prensa y archivo multimedia. La tónica general es que la búsqueda se remonta hasta el año 2004 y es de carácter cronológico. Solamente en tres casos hemos encontrado la opción de búsqueda cronológica acompañada por una opción de búsqueda de palabras en el campo título o texto de la nota de prensa. Sirvan de muestra los buscadores de notas de prensa del Ministerio de Cultura, del Ministerio de Asuntos Exteriores y de Cooperación, y del Ministerio de Medio Ambiente y Medio Rural y Marino.

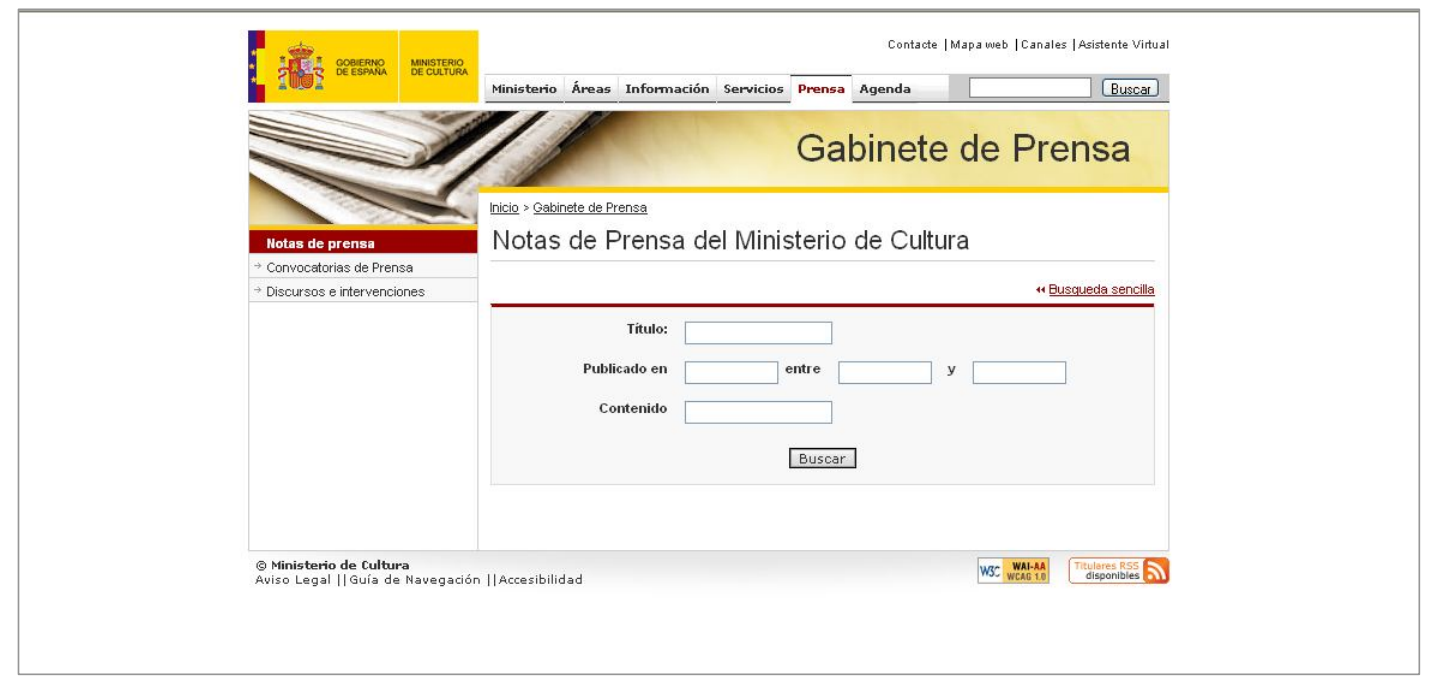

Figura 3. Buscador de Notas de Prensa. Gabinete de Prensa del Ministerio de Cultura. Fuente: Sección web del Ministerio de Cultura. Búsqueda en las Notas de Prensa. Gabinete de Prensa. 
Portales para la comunicación estratégica de las instituciones políticas

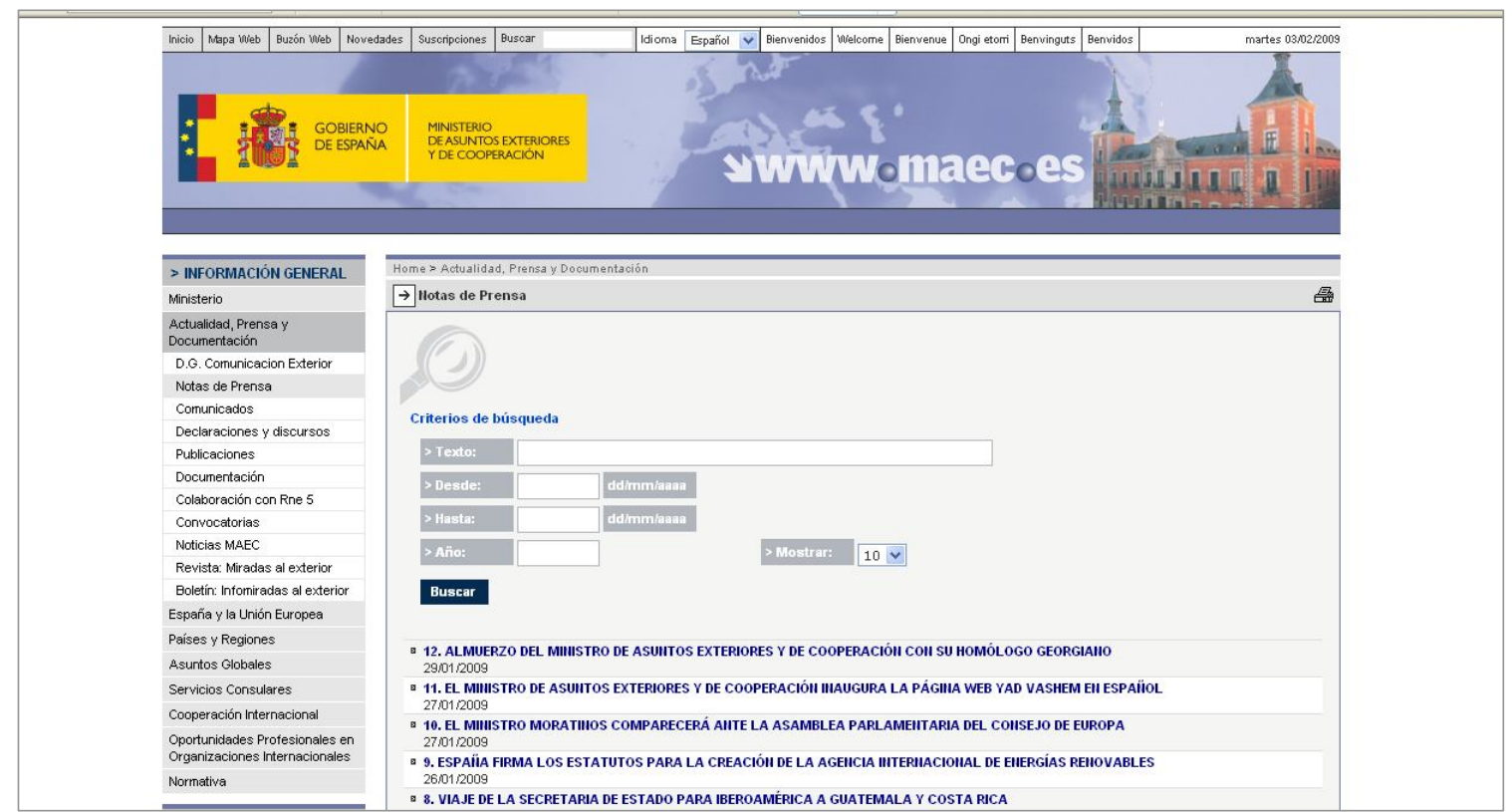

Figura 4. Buscador de Notas de Prensa. Gabinete de Prensa del Ministerio de Asuntos Exteriores y de Cooperación.

Fuente: Sección web del Ministerio de Asuntos Exteriores y de Cooperación. Búsqueda en las Notas de Prensa. Gabinete de Prensa.

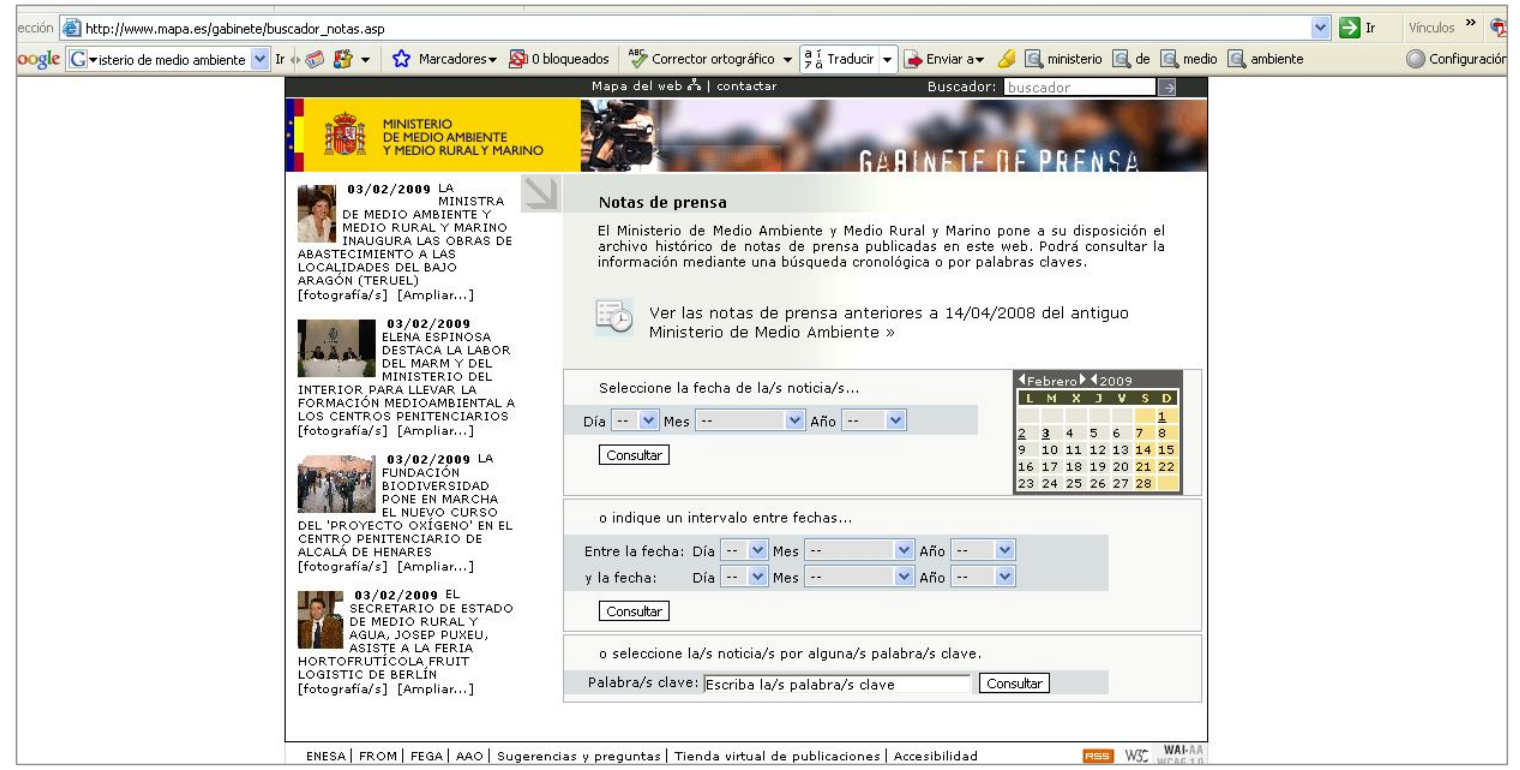

Figura 5. Buscador de Notas de Prensa. Gabinete de Prensa del Ministerio de Medio Ambiente y Medio Rural y Marino.

Fuente: Sección web del Ministerio de Medio Ambiente y Medio Rural y Marino. Búsqueda en las Notas de Prensa. Gabinete de Prensa. 
Victoria Martínez Navarro/Mª Dolores Ayuso García

Si ahondamos un poco más en el análisis de los resultados de este indicador evaluado podemos afirmar que la mayoría de las fuentes de información de estas sedes datan de la VIII Legislatura. En concreto de marzo de 2004, tras la victoria en las elecciones generales del Partido Socialista Obrero Español, la consulta de las fuentes de información de legislaturas inmediatamente anteriores (en las que gobernaba el Partido Popular) no resulta viable desde las web ministeriales. Por tanto, nos encontramos ante unas fuentes de información de actualidad, cuyo retrospectivo más exhaustivo data del inicio de la legislatura del partido en el poder en el momento de llevar a cabo esta investigación. Esta realidad condiciona el uso de dichas fuentes para el denominado periodismo de investigación, o mejor dicho prácticamente las invalida.

\section{Conclusiones.}

Las nuevas tecnologías tienen un efecto de regeneración de la democracia. La actividad política del día llega al ciudadano a través de las web ministeriales para intentar movilizar a usuarios que están menos expuestos y menos predispuestos a los mensajes propagandísticos canalizados por los medios tradicionales. El hecho de que los medios digitales permitan llegar a la ciudadanía a un coste bajo y que además, logren segmentar los mensajes que el líder político quiera enviar son las grandes ventajas.

La ventaja competitiva en los casos analizados alude al hecho de que ésta se genera cuando las instituciones política son capaces de aprovechar de manera eficaz aquellos recursos y habilidades únicos e inimitables que les permiten crear más valor que sus competidores, podemos asumir que la comunicación corporativa como parte de la estrategia de marketing de las organizaciones contribuye a generar una ventaja competitiva, puesto que mediante las diversas acciones de comunicación corporativa o institucional la institución puede incrementar el valor de sus actividades y servicios en el mercado, llamando así la atención sobre algunos atributos y funciones que pudieran estar siendo ignorados por los ciudadanos.

Las estrategias que hemos estudiado a lo largo de este trabajo ponen claramente de manifiesto que el mensaje político se ha adecuado a los códigos de Internet, que requieren de rapidez, accesibilidad, viveza y de un lenguaje directo y mucho más visual. Las nuevas tecnologías forman parte de las herramientas para la comunicación estratégica del partido político que ostenta el poder gubernamental. De ahí que podamos afirma tras este análisis de 
Portales para la comunicación estratégica de las instituciones políticas

la situación que la Red se ha transformado en el principal aliado para que las instituciones políticas de un país se acerquen a sus habitantes.

\section{Bibliografía.}

Ayu so García, MDolores y Martínez Navarro, Victoria: "Gobierno electrónico. Contenidos y organización de las sedes web de los parlamentos autonómicos". Revista española de documentación científica. vol. 28, nº 4, 2005, p. 462-478.

Canel, María José (2006): Comunicación política. Una guía para su estudio y práctica. Madrid: Tecnos.

. (2007): Comunicación de las instituciones públicas. Madrid: Tecnos.

Del Pozo Lite, Marisa (1997): Cultura empresarial y comunicación interna : Su influencia en la gestión estratégica. Madrid: Fragua.

Hernández Alonso, Francisco J. (2008): "La política al servicio de los medios de comunicación”. En Cuenta y Razón. No 4, p. 43-50.

García Beaudoux, Virginia; D’Adamo, Orlando y Slanvinsky, Gabriel (2007): Comunicación política y campañas electorales : estrategias en elecciones presidenciales. Barcelona : Gedisa.

Libro blanco sobre una política europea de comunicación :(presentado por la Comisión) Bruselas, 01,02,2006 COM (2006) 35 final. -- Luxemburgo : Oficina de Publicaciones Oficiales de las Comunidades Europeas.

Martínez Nicolás, Manuel (2007): “Agitación en el campo. Nueve ideas para la investigación sobre comunicación política en España”. En Política y sociedad. Vol. 44, N² 2, p. 209-227.

Monzón, Cándido (2006): Opinión pública, comunicación y política. Madrid : Tecnos. 
Sánchez Tabernero, Alfonso (2000): Dirección estratégica de empresas de comunicación. Madrid: Cátedra.

Torregrosa Carmona, Francisco y García Jiménez, Antonio (2007): “Una aproximación a la documentación en la comunicación política”. En Documentación de las ciencias de la información. № 30, p. 315-327. 
Portales para la comunicación estratégica de las instituciones políticas

\section{Anexo I. Url's sedes web Ministeriales. Administración General del Estado Español.}

Ministerio de la Presidencia [en línea]. [fecha de acceso 16 de septiembre 2007]. Disponible en Internet: <http://www.mpr.es>.

Ministerio de Economía y Hacienda. [en línea] . [fecha de acceso 16 de septiembre 2007]. Disponible en Internet: <http://www.meh.es>.

Ministerio de Asuntos Exteriores y Cooperación. [en línea] . [fecha de acceso 16 de septiembre 2007]. Disponible en Internet: 〈http://www.maec.es>.

Ministerio de Justicia. [en línea] . [fecha de acceso 16 de septiembre 2007]. Disponible en Internet: <http://www.mjusticia.es>.

Ministerio de Defensa. [en línea] . [fecha de acceso 16 de septiembre 2007]. Disponible en Internet: 〈http://www.mde.es>.

Ministerio del Interior. [en línea] . [fecha de acceso 16 de septiembre 2007]. Disponible en Internet: <http://www.mir.es>.

Ministerio de Fomento. [en línea] . [fecha de acceso 16 de septiembre 2007]. Disponible en Internet: <http://www.fomento.es>.

Ministerio de Educación, Política Social y Deporte. [en línea] . [fecha de acceso 16 de septiembre 2007]. Disponible en Internet: <http://www.mepsyd.es>.

Ministerio de Trabajo e Inmigración. [en línea] . [fecha de acceso 16 de septiembre 2007]. Disponible en Internet: <http://www.mtas.es>.

Ministerio de Industria, Turismo y Comercio. [en línea] . [fecha de acceso 16 de septiembre 2007]. Disponible en Internet: <http://www.mityc.es>

Ministerio de Medio Ambiente, Medio Rural y Marino. [en línea] . [fecha de acceso 16 de septiembre 2007]. Disponible en Internet: 〈http://www. marm.es>. 
Ministerio de Administraciones Públicas. [en línea] . [fecha de acceso 16 de septiembre 2007]. Disponible en Internet: <http://www.map.es>.

Ministerio de Cultura. [en línea] . [fecha de acceso 16 de septiembre 2007]. Disponible en Internet: <http://www.mcu.es>.

Ministerio de Sanidad y Consumo. [en línea] . [fecha de acceso 16 de septiembre 2007].

Disponible en Internet: 〈http://www.msc.es>.

Ministerio de Vivienda. [en línea] . [fecha de acceso 16 de septiembre 2007]. Disponible en Internet: <http://www.mviv.es>.

Ministerio de Ciencia e Innovación. [en línea] . [fecha de acceso 16 de septiembre 2007].

Disponible en Internet: <http:// web.micinn.es>.

Ministerio de Igualdad. [en línea] . [fecha de acceso 16 de septiembre 2007]. Disponible en Internet: <http://www.migualdad.es>. 